\title{
OSTEOGENIC DIFFERENTIATION AS A RESULT OF BMP-2 PLASMID DNA BASED GENE THERAPY IN VITRO AND IN VIVO
}

\author{
F. Wegman ${ }^{1}$, A. Bijenhof ${ }^{1}$, L. Schuijff ${ }^{1}$, F.C. Öner ${ }^{1}$, W.J.A. Dhert ${ }^{1,2}$ and J. Alblas ${ }^{1 *}$ \\ ${ }^{1}$ Department of Orthopaedics, University Medical Center Utrecht, The Netherlands \\ ${ }^{2}$ Faculty of Veterinary Medicine, Utrecht University, The Netherlands
}

\begin{abstract}
Bone regeneration is one of the major focus points in the field of regenerative medicine. A well-known stimulus of bone formation is bone morphogenetic protein-2 (BMP2), which has already been extensively used in clinical applications. We investigated the possibility of achieving osteogenic differentiation both in vitro and in vivo as a result of prolonged presence of BMP-2 using plasmid DNA-based gene therapy. By delivering BMP-2 cDNA in an alginate hydrogel, a versatile formulation is developed. High transfection efficiencies of up to $95 \%$ were obtained in both human multipotent stromal cells (MSCs) and MG-63 cells using naked DNA in vitro. Over a period of 5 weeks, an increasing amount of biologically active BMP-2 was released from the cells and remained present in the gel. In vivo, transfected cells were found after both two and six weeks implantation in naked mice, even in groups without seeded cells, thus indicating in vivo transfection of endogenous cells. The protein levels were effective in inducing osteogenic differentiation in vitro, as seen by elevated alkaline phosphatase (ALP) production and in vivo, as demonstrated by the production of collagen I and osteocalcin in a mineralised alginate matrix.

We conclude that BMP-2 cDNA incorporated in alginate hydrogel appears to be a promising new strategy for minimal-invasive delivery of growth factors in bone regeneration.
\end{abstract}

Keywords: Bone morphogenetic protein (BMP), osteogenic differentiation, multipotent stromal cells (MSC), transfection, hydrogel, bone graft, bone regeneration.

\author{
*Address for correspondence: \\ Jacqueline Alblas \\ Department of Orthopaedics, Room G05.228, \\ University Medical Center Utrecht, \\ P.O. Box 85500, \\ 3508 GA Utrecht, \\ The Netherlands
}

Telephone Number: +31-88-7550031 FAX Number:: +31-30-2510638

E-mail: J.Alblas@umcutrecht.nl

\section{Introduction}

Bone regeneration is one of the major focus points within the field of regenerative medicine. To create bone tissue and augment defects, many strategies have been used, such as different cell based strategies, material development and growth factor delivery (Khan et al., 2008; Arthur et al., 2009).

An important growth factor in bone tissue engineering is bone morphogenetic protein 2 (BMP-2), member of the transforming growth factor $\beta$ (TGF- $\beta$ ) superfamily. BMP-2 is involved in committing multipotent stromal cells (MSCs) towards the osteogenic lineage and known to induce new bone formation (Riley et al., 1996). Both in vitro and in vivo BMP-2 has shown strong osteoinductive activity. However, large amounts of rhBMP-2 protein are administered clinically for an osteogenic effect mainly due to fast degradation by proteinases. Animal studies have shown a half-life of 7-16 min systemically, and up to 8 days locally when implanted on a collagen sponge (Poynton and Lane, 2002). The possible negative side effects for patients due these high doses of BMP-2 may result in vertebral osteolysis, ectopic bone formation, radiculitis and cervical soft tissue swelling (Shimer et al., 2009).

BMP-2 gene therapy is an alternative that might be able to overcome the use of high doses of BMP-2, because when applied to cells a relatively sustained delivery of protein can be achieved (Southwood et al., 2004). Gene therapy can be performed by viral or non-viral DNA delivery. Non-viral has several advantages over viral gene therapy, the latter one resulting in high transfection efficiencies but associated with problems such as immunogenicity, virus-dependent recombination risks and the protein expression can exceed the timeframe required for bone defect healing (Luo and Saltzman, 2000; Louise, 2006; Pietersz et al., 2006). Non-viral gene delivery is considered much safer and usually provides a transient expression of the desired gene. However many approaches result in a low transfection efficiencies. Therefore much effort is invested into the development of an efficient nonviral gene delivery method, with the use of various transfection agents (Pelled et al., 2010). Particularly well studied are liposomes, phospholipid bilayer vesicles which can easily merge with the cell membrane to deliver encapsulated plasmid DNA (Audouy et al., 2002; Dass, 2004; Ma et al., 2007).

Another carrier for gene delivery, which is compatible with simultaneous cell seeding, is alginate hydrogel. Alginate is an anionic linear copolymer of $\beta$-Dmannuronic acid and $\alpha$-L-glucuronic acid residues that gelates with bivalent cations like $\mathrm{Ca}^{2+}$ (Kong et al., 2008). 
It is FDA approved, non-toxic, biocompatible and extensively used in the food and drug industry. It has been used as a vehicle for plasmid DNA in the form of nanoparticles or combined with other hydrogels (Krebs et al., 2010; Park et al., 2007) and was applied in bone tissue engineering applications both in vitro and in vivo (Stevens et al., 2005; Hunt and Grover, 2010). This makes alginate very suitable both as gene activated matrix (GAM), and for cell seeded constructs.

Our aim is to develop an injectable hydrogel for bone regeneration, containing different biological components, i.e., cells and BMP-2 cDNA, which are beneficial for bone formation in a specific setting. The present study investigates the transfection efficiency of epitope-tagged BMP-2 plasmid DNA in primary human MSCs and the MG-63 cell line in alginate, and the amount of protein produced and released from the gel in vitro as well as its bioactivity. To investigate the contribution of each of the components to scaffold performance, the components are tested separate and combined. A cell-free construct was included to investigate whether resident cells at the implantation site could express functional BMP-2 from the GAM.

\section{Materials and Methods}

\begin{abstract}
Alginate gel
Autoclaved high-viscosity non-medical-grade alginate powder (International Specialty Products, ISP, Memmingen, Germany) was dissolved at a concentration of $20 \mathrm{mg} / \mathrm{ml}$ in alpha minimum essential medium ( $\alpha$-MEM; Gibco, Breda, The Netherlands). The gel was polymerised by adding an equal volume of $100 \mathrm{mM}$ autoclaved $\mathrm{CaCl}_{2}$ supplemented with $10 \mathrm{mM}$ of 4-(2-hydroxyethyl)-1piperazineethanesulfonic acid (HEPES) pH 7.4 (Gibco) for $10 \mathrm{~min}$. Ca-solution was then replaced by $1 \mathrm{ml}$ culture medium.
\end{abstract}

\section{Cell culture}

MG-63 cells (osteosarcoma cell-line, ATCC \#CRL-1427; ATCC, Wesel, Germany) were cultured in proliferation medium consisting of Dulbecco's modified Eagle's Medium (Gibco) supplemented with $15 \%$ (v/v) newborn calf serum (Gibco), $100 \mathrm{U} / \mathrm{ml}$ penicillin and $100 \mu \mathrm{g} / \mathrm{ml}$ streptomycin (Gibco).

Human MSCs were isolated from bone marrow, aspirated from the iliac crest or acetabulum of patients after their informed consent and collected in heparin coated tubes. The mononuclear fraction was isolated using Ficoll density gradient centrifugation. The MSCs are isolated by adherence to tissue culture plastic, cultured in aMEM, supplemented with $10 \%$ (v/v) foetal calf serum (Cambrex, Charles City, IA, USA), $100 \mathrm{U} / \mathrm{ml}$ penicillin, $100 \mu \mathrm{g} / \mathrm{ml}$ streptomycin, $0.2 \mathrm{mM}$ L-ascorbic acid-2-phosphate (AsAP) (Sigma-Aldrich, St. Louis, MO, USA) and $1 \mathrm{ng} /$ ml FGF-2 (R\&D Systems, Minneapolis, MN, USA).

Goat MSCs were isolated similarly from bone marrow (BM), aspirated from the iliac wings of Dutch milk goats and cultured in $\alpha$ MEM (Gibco), supplemented with $15 \%$ (v/v) foetal calf serum (Cambrex), $100 \mathrm{U} / \mathrm{ml}$ penicillin, $100 \mu \mathrm{g} / \mathrm{ml}$ streptomycin and $2 \mathrm{mM}$ L-glutamine
(Glutamax, Gibco). All cells were maintained at $37^{\circ} \mathrm{C}$ and $5 \% \mathrm{CO}_{2}$ in a humidified incubator.

\section{Transfection procedures}

For expression of BMP-2 the plasmid pcDNA3.1/His/ hBMP-2 was used, in which full-length human recombinant BMP-2 is in frame preceded by a 18 nucleotide polyhistidine region (His tag). This tag is used to detect transfected cells and discriminate between endogenous BMP-2 and BMP-2 produced as a result of transfection. During secretion of BMP-2 from cells, the tag may be removed together with the signal sequence.

Plasmid DNA (0-50 $\mu \mathrm{g} / \mathrm{ml}$ of pcDNA3.1/His/hBMP2 or pEGFP-N1(BD Biosciences, Franklin Lakes, NJ, USA))/ Lipofectamine2000 (Invitrogen, Breda, The Netherlands) complexes were prepared according to the manufacturer's instructions. Per sample, the DNA, either or not complexed with liposomes and $10^{6}$ trypsinised cells was mixed with $50-100 \mu \mathrm{l}$ of alginate hydrogel $(20 \mathrm{mg} /$ $\mathrm{ml}$ ). This gel mixture was polymerised by adding 100$200 \mu \mathrm{l} \mathrm{CaCl} / \mathrm{HEPES}$ solution. After $10 \mathrm{~min}$, supernatant was removed and replaced with culture medium. The gels were cultured at $37^{\circ} \mathrm{C}$ and $5 \% \mathrm{CO}_{2}$ in a humidified incubator for up to 5 weeks before analysis of gene expression. For preparation of the in vivo implants, see below.

\section{FACS analysis of BMP-2, ALP and GFP}

Constructs of $100 \mu \mathrm{l}$ gel containing plasmid DNA (pcDNA3.1/His/hBMP-2) and $10^{6}$ cells were depolymerised using citrate buffer $(150 \mathrm{mM} \mathrm{NaCl}, 55 \mathrm{mM}$ sodium citrate and $20 \mathrm{mM}$ EDTA in $\mathrm{H}_{2} \mathrm{O}$ ) for $15 \mathrm{~min}$ at $37^{\circ} \mathrm{C}$. Cells were counted and $90-110 \%$ of the seeded cells were retrieved. Cells were pelleted and fixed in $4 \%(\mathrm{v} / \mathrm{v})$ formalin (Clinipath, Duiven, The Netherlands) in PBS for 10 min. All subsequent washing steps were done with PBS/ $1 \%(\mathrm{w} / \mathrm{v}) \mathrm{BSA}$. The cells were washed with PBS/BSA three times and permeabilised with $0.1 \%$ (v/v) Triton-X100 (Fluka, Zwijndrecht, The Netherlands) in PBS for $10 \mathrm{~min}$. The cells were washed again and incubated with $2 \mu \mathrm{g} / \mathrm{ml}$ anti-His (recognizing His-tagged BMP-2) antibody (Genscript, Piscataway, NJ, USA) in PBS/BSA for $1 \mathrm{~h}$ at room temperature (RT). Control samples were incubated with a mouse isotype-matched control $\operatorname{IgG}(2 \mu \mathrm{g} / \mathrm{ml})$ (Dako, Glostrup, Denmark). After antibody incubation, cells were washed with PBS/BSA and incubated in the dark with the secondary antibody goat-anti-mouseAlexa488 (Invitrogen), diluted to $20 \mu \mathrm{g} / \mathrm{ml}$ in PBS/BSA for $30 \mathrm{~min}$ at RT. After secondary antibody incubation cells were washed and taken up in PBS/BSA and analysed for green fluorescence by FACS Calibur. For ALP analysis the same procedure was used, with the primary antibody B4-78-C (Developmental Studies Hybridoma Bank of Iowa, Iowa City, IA, USA) diluted 1:100 in PBS/BSA and incubated for $1 \mathrm{~h}$ at RT, followed by incubation in the dark for $30 \mathrm{~min}$ at RT with the secondary antibody goat-antimouse-PE (Southern Biotech, Birmingham, AL, USA), diluted to $0.25 \mu \mathrm{g} / \mathrm{ml}$ in PBS/BSA. For GFP transfected cells, a similar cell isolation from gel was performed and cells were analysed for GFP intensity at day 7 (optimal expression of GFP in alginate) using the FACS Calibur. 


\section{BMP-2 ELISA}

Constructs of $100 \mu \mathrm{l}$ gel were cultured for up to 5 weeks and medium $(2 \mathrm{ml})$ was changed weekly. The collected medium was stored at $-20^{\circ} \mathrm{C}$. Gel samples were prepared by depolymerising alginate with citrate buffer for 15 minutes at $37^{\circ} \mathrm{C}$. Cells were centrifuged and the supernatant containing the dissolved protein was stored at $-20^{\circ} \mathrm{C}$. The ELISA (Quantikine \#DBP200 from R\&D Systems) was performed on thawed samples following the standard protocol.

\section{Preparation in vivo implants}

Six different constructs of $300 \mu$ l alginate $(10 \mathrm{mg} / \mathrm{ml})$ gel each were constructed and polymerised with $1 \mathrm{ml}$ of 100 $\mathrm{mM}$ autoclaved $\mathrm{CaCl}_{2}$, supplemented with $10 \mathrm{mM}$ of 4(2-hydroxyethyl)-1-piperazineethanesulfonic acid (HEPES) pH 7.4 (Gibco), for $10 \mathrm{~min}$. One group consisted of only alginate, one group contained $3 \mu \mathrm{g}$ of His/BMP-2 plasmid DNA in alginate, four groups consisted of alginate with $3 \mu \mathrm{g}$ His/BMP-2 plasmid DNA combined with 3 million MSCs from 4 different donors. The latter four contained cells from two human MSC donors, or two goat MSC donors. The gels were implanted immediately after polymerisation.

\section{Animals and implantation}

18 female nude mice (Hsd-cpb:NMRI-nu, Harlan, Boxmeer the Netherlands) were anaesthetised with $1.5 \%$ isoflurane, after which the implants were placed in 4 separate subcutaneous dorsal pockets per mouse $(n=6$ per group / time point). The animals were postoperatively treated with the analgesic buprenorphine $(0.05 \mathrm{mg} / \mathrm{kg}, \mathrm{sc}$; Temgesic, Schering-Plough/Merck, Whitehouse Station, NJ, USA) and housed together at the Central Laboratory Animal Institute, Utrecht University. Experiments were conducted with the permission of the local Ethical Committee for Animal Experimentation and in compliance with the Institutional Guidelines on the use of laboratory animals.

\section{Explantation and embedding}

Two and six weeks after implantation the constructs were retrieved to analyse transfection, osteogenic differentiation and alginate properties in vivo. Samples were fixed overnight in $4 \%$ (v/v) formalin containing $100 \mathrm{mM} \mathrm{CaCl}_{2}$ and processed for $5 \mu \mathrm{M}$ thick paraffin sections through alcohol dehydration series.

\section{Histology}

To analyse the alginate properties in vivo and investigate blood vessel formation, haematoxylin/eosin (HE) stainings were performed on all samples. Samples were scored on morphology, calcification and blood vessel ingrowth by two observers. A scale of 0-2 was applied; 0 indicating absence of the readout parameter, 1 for some morphology changes, calcification or blood vessel presence, and 2 for significant/abundant presence. To detect His/BMP-2transfected cells in the implants, anti-His immunohistochemistry was performed. Sections were permeabilised with Triton X-100 and blocked in $3 \% \mathrm{H}_{2} \mathrm{O}_{2}$ for $10 \mathrm{~min}$ and $5 \% \mathrm{BSA}$ for $30 \mathrm{~min}$. Antigen retrieval steps were performed using $1 \mathrm{mg} / \mathrm{ml}$ pronase and $10 \mathrm{mg} / \mathrm{ml}$ hyaluronidase for $30 \mathrm{~min}$ each. Sections were subsequently incubated with $5 \mu \mathrm{g} / \mathrm{ml}$ biotinylated anti-His antibody (Genscript, Piscataway, NJ, USA, A00613) for $1 \mathrm{~h}$ and as second antibody $3.3 \mu \mathrm{g} / \mathrm{ml}$ streptavidin/ horseradish peroxide (HRP) was used. For collagen I, a similar protocol was performed using rabbit-anti-collagen I primary antibody (Abcam, Cambridge, UK; Ab34710) at $3.3 \mu \mathrm{g} /$ $\mathrm{ml}$ and a goat-anti-rabbit-streptavidin/HRP (Invitrogen, 624320)) secondary antibody at $6.6 \mu \mathrm{g} / \mathrm{ml}$. For osteocalcin, a similar protocol was performed using a rabbit-antiosteocalcin primary antibody (Alexis ALX-210-333; now Thermo Fisher Scientific, Waltham, MA, USA) at $2 \mu \mathrm{g} /$ $\mathrm{ml}$ and a goat-anti-rabbit-streptavidin/HRP (Invitrogen) secondary antibody at $6.6 \mu \mathrm{g} / \mathrm{ml}$. All stainings were developed with diaminobenzidine (DAB) and Mayer's haematoxylin was used for counterstaining. A double staining was also performed using the same primary antibodies and concentrations but with fluorescent secondary antibodies. First the collagen I antibody was added to samples for $1 \mathrm{~h}$ and after several wash steps the matching goat-anti-rabbit-Alexa488 (Invitrogen, A52700) at $13.3 \mu \mathrm{g} / \mathrm{ml}$ was incubated. Then $5 \mu \mathrm{g} / \mathrm{ml}$ biotinylated anti-His antibody was added for $1 \mathrm{~h}$, followed by antibiotin streptavidin/Alexa594 (Molecular Probes, Eugene, OR, USA; S11227) at $6.6 \mu \mathrm{g} / \mathrm{ml}$ for $30 \mathrm{~min}$. All antibodies were diluted in PBS/BSA.

\section{Von Kossa staining}

Sections were deparaffinised and incubated with a $1 \%$ (w/ v) silver nitrate solution (Fisher Scientific) under a 60$100 \mathrm{~W}$ light bulb for $1 \mathrm{~h}$. After washing with distilled water, unreacted silver is removed by $5 \%(\mathrm{w} / \mathrm{v})$ sodium thiosulphate solution for $5 \mathrm{~min}$. Slides were counterstained with nuclear Fast Red.

\section{Alizarin Red staining}

Sections were covered for 1-2 min with $2 \%$ (w/v) Alizarin Red (Merck, Darmstadt, Germany) and $0.5 \%$ (v/v) ammonium hydroxide in distilled water, final $\mathrm{pH}$ 4.0. After incubation sections were washed with distilled water and mounted in Depex.

\section{Goldner's trichrome staining}

Sections were deparaffinised and incubated with haematoxylin (Weigert's) for $5 \mathrm{~min}$. Sections were washed with tap water for 5 min and incubated with Goldner's 1 solution, containing $0.2 \%$ (v/v) glacial acetic acid, $0.033 \%$ $(\mathrm{w} / \mathrm{v})$ acid fuchsine and $0.13 \%(\mathrm{w} / \mathrm{v})$ Ponceaux de xilidine (Fluka), for 30-45 min. After rinsing with $1 \%(\mathrm{v} / \mathrm{v})$ acetic acid, sections were stained with Orange G (Fluka, 75380) for $7 \mathrm{~min}$ and washed again with $1 \%$ acetic acid. Sections were counterstained with Light green (Sigma-Aldrich, L5382) for $7 \mathrm{~min}$ and after rinsing with $1 \%$ acetic acid were dehydrated and mounted in Depex.

\section{Safranin O staining}

Sections were deparaffinised and incubated with haematoxylin (Weigert's) for $5 \mathrm{~min}$. After washing in running tap water, sections were dipped in distilled water and counterstained in $0.4 \%$ (w/v) Fast Green solution. 


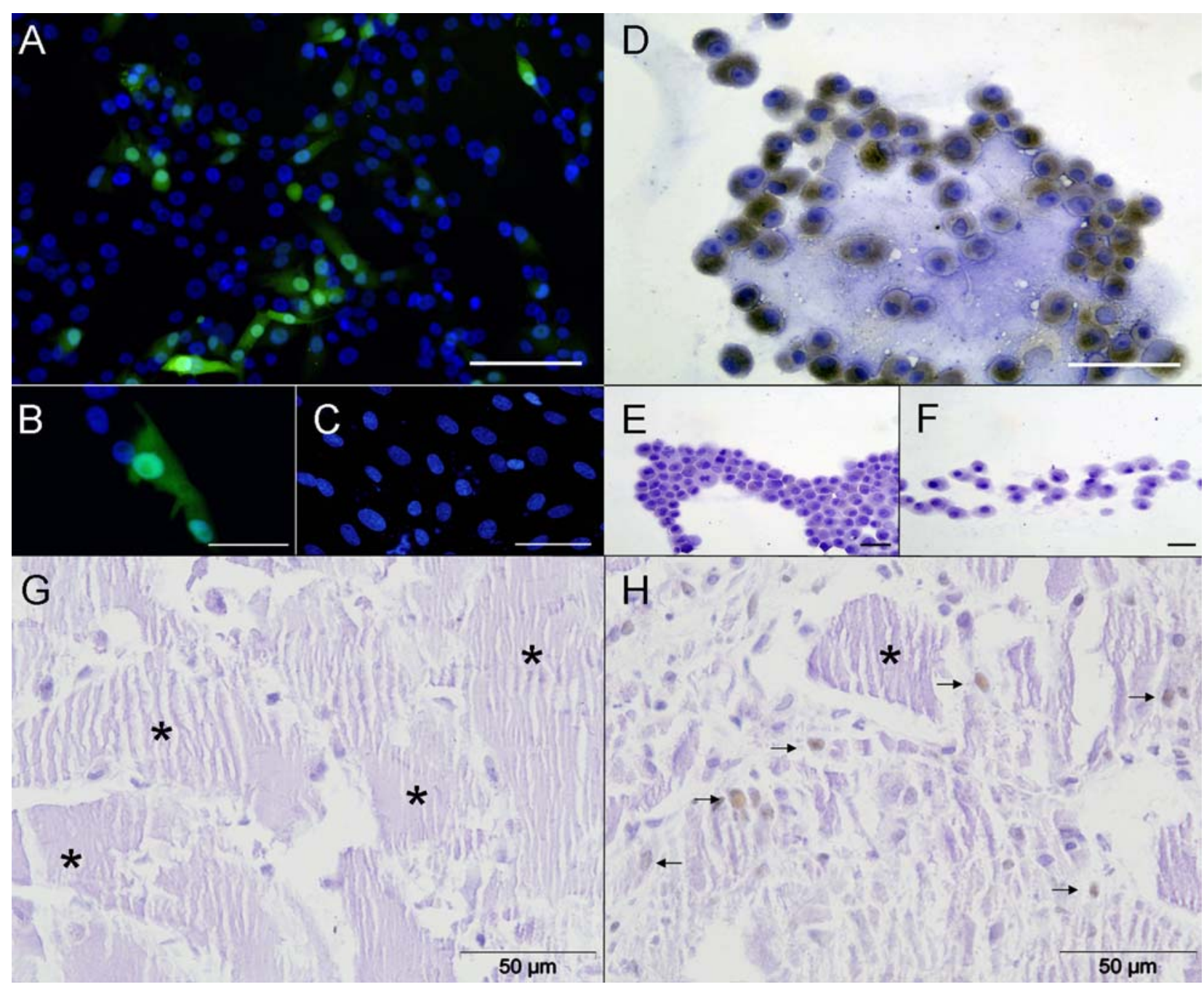

Fig. 1. The alginate delivery system: GFP and BMP-2 transfected cells in vitro and in vivo. (A, B) GFP transfected, (C) Untransfected, (D) His-tagged BMP-2 transfected, $\alpha$-His staining, (E) His-tagged BMP-2 transfected, isotypematched control Ab staining, (F) Untransfected control, $\alpha$-His staining. Pictures A-F are representative of 3 independent experiments on MG-63 cells, performed in duplicate. (G) Anti-His staining of cell-free untransfected sample after 6 weeks in vivo. (H) Anti-His staining of hMSCs + BMP-2 plasmid DNA sample after 6 weeks in vivo. Panels $\mathbf{G}$ and $\mathbf{H}$ are representative for 6 implants. Arrows indicate transfected cells, *marks alginate, scale bars represent $50 \mu \mathrm{m}$.

Sections were rinsed in $1 \%(\mathrm{v} / \mathrm{v})$ acetic acid until colour no longer runs and counterstained again in freshly prepared $0.125 \%$ (w/v) Safranin O (Merck) staining. After short dehydration protocol, sections were mounted in Depex.

\section{Statistics}

The statistical significance of differences between experimental groups in Figure 5 was assessed using a Fisher exact test for presence/absence of collagen I. Multiple comparisons were generated using a Bonferroni correction to calculate the $p$-values. A $p$-value of $<0.05$ was considered significant.

\section{Results}

We developed an alginate gene delivery system for use with osteoblasts and their precursors, including MSCs. To show that cell transfection in alginate is an effective method, the tracer gene enhanced green fluorescent protein (EGFP) was transfected in hMSCs or MG-63 cells, either or not after complexation with liposomes. After 7 days, the transfected cells and untransfected controls were isolated from the alginate and analysed for GFP expression. On DAPI counterstained cytospins, the positive cells were scored under a fluorescence microscope, resulting in transfection efficiencies ranging from $35-50 \%$ (Fig. 1A, B). For further quantification, FACS analysis was performed on transfected and untransfected cells. Cells were analysed for their fluorescence intensity level; the cells were considered transfected at a fluorescence intensity level that excluded $95 \%$ of the untransfected cells. To compare the average amount of protein produced/cell between different groups, the mean fluorescence intensity (MFI) was analysed. For GFP this resulted in a MFI of 156-157 compared to 25 for the control (Fig. 2A). The transfection efficiency was confirmed for both the liposome-complexed group and the naked plasmid DNA group. 

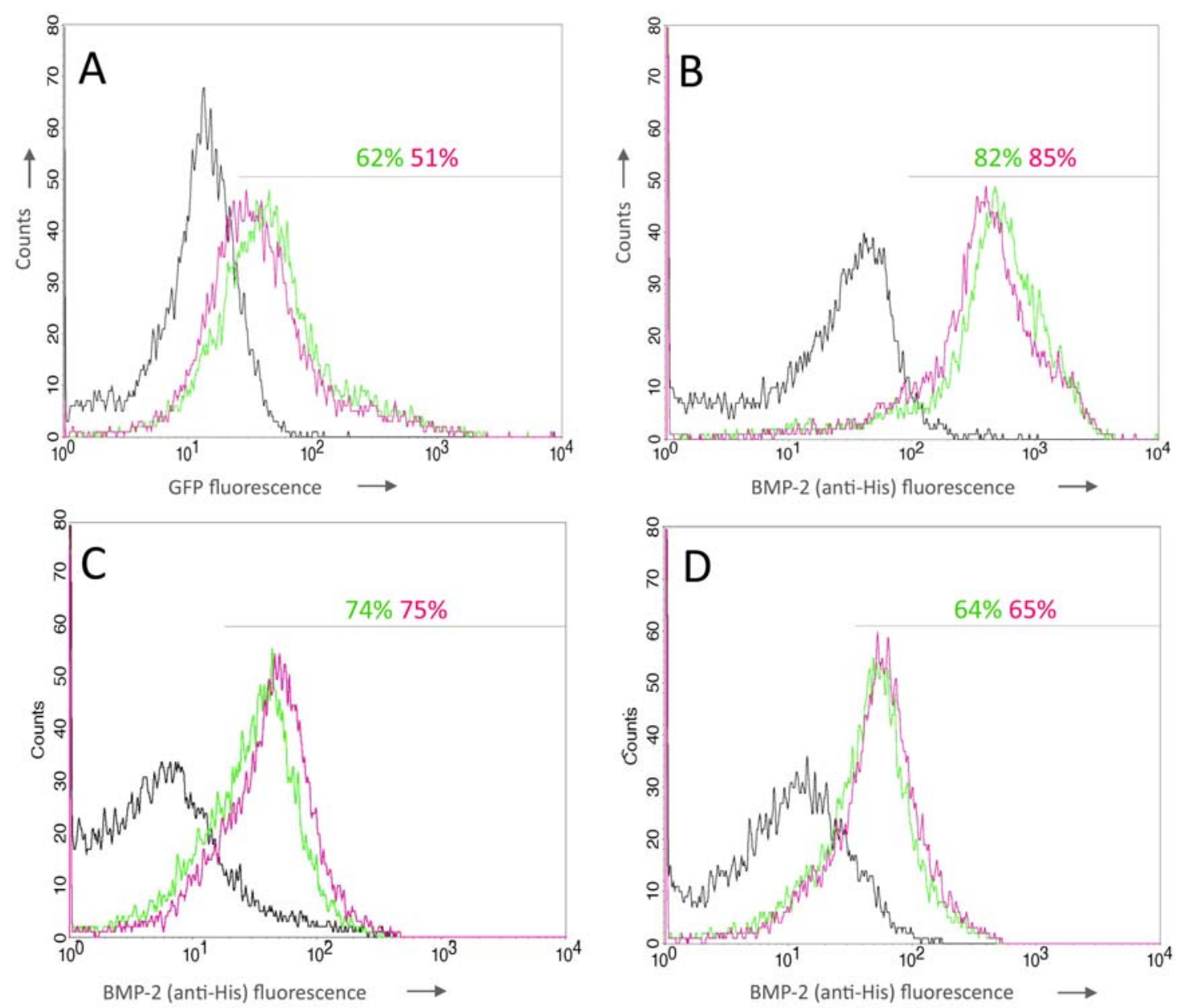

Fig. 2. FACS analysis of GFP- or His/BMP-2 transfection efficiency of MSCs and MG-63 cells in alginate. (A) GFP fluorescence of untransfected MG-63 (black line), GFP transfection with liposomes (green line), GFP transfection without liposomes (pink line). (B, C, D) anti-His staining of His/BMP-2 transfected MG-63 cells (B), goat MSCs (C) or human MSCs (D) with liposomes (green line) or without liposomes (pink line); staining of transfected cells with isotype-matched control antibody (black line). The horizontal black region excludes $95 \%$ of the control cells. Graphs are representative of 3 independent experiments performed in duplicate.

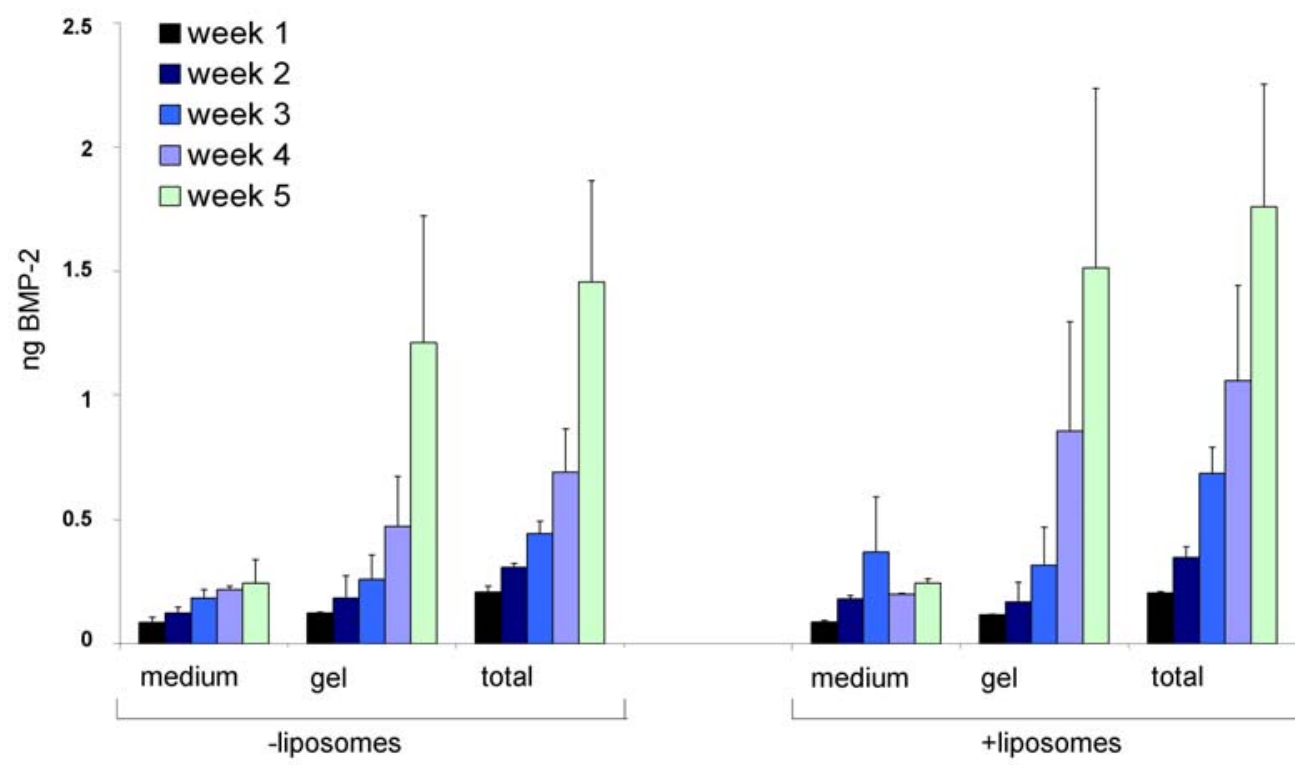

Fig. 3. rhBMP-2 release/week from transfected MG-63 cells in alginate. BMP-2 release/week from $0.1 \mathrm{ml}$ alginate constructs containing 1 million cells and either naked (-lipo) or liposome complexed (+lipo) His/BMP-2 plasmid DNA. BMP-2 release was analysed in two independent experiments in duplicate in the medium and in the gel separately by ELISA. The results represent mean $\pm \mathrm{SD}$. 
A
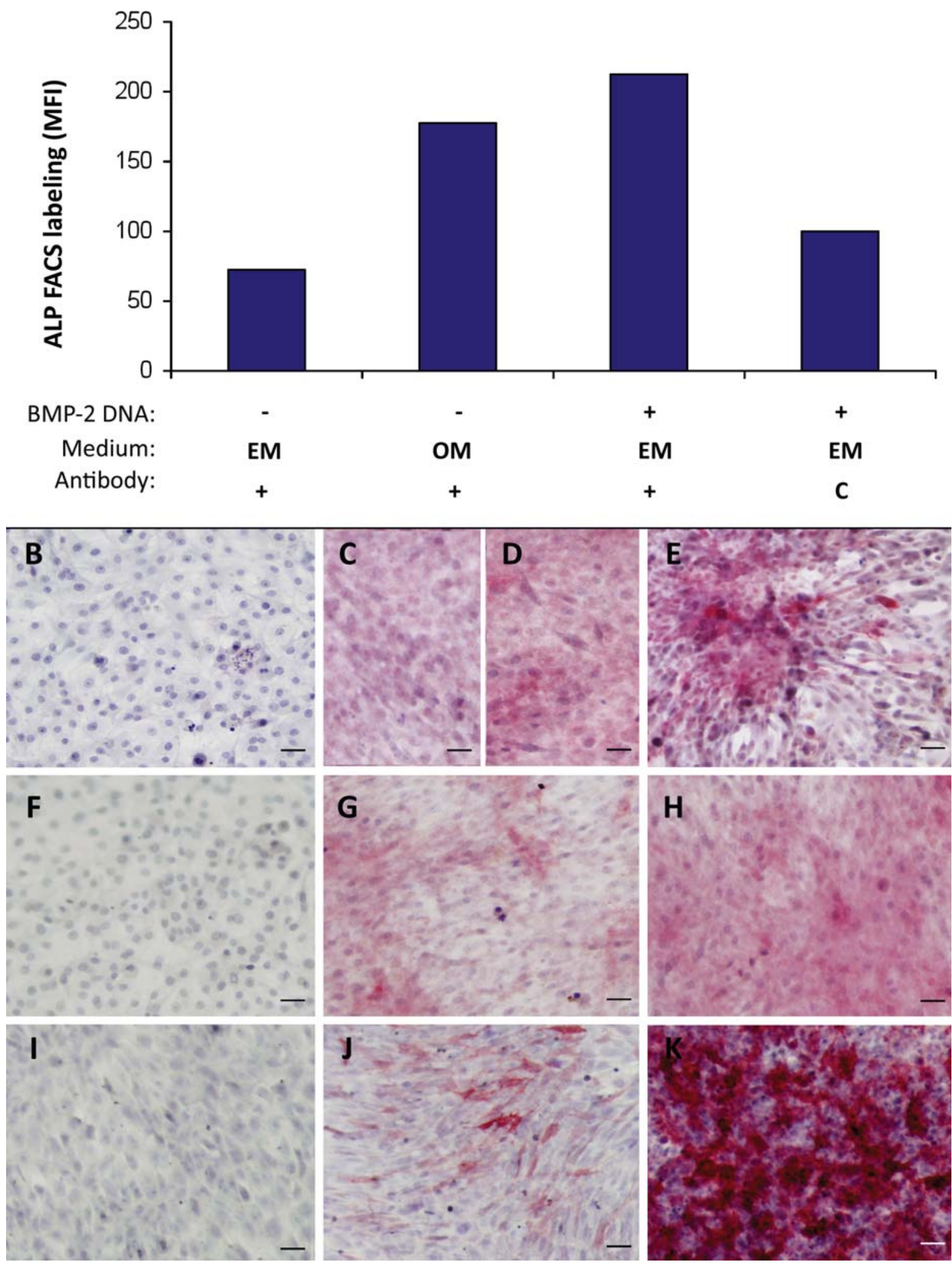

Fig. 4. Alkaline phosphatase expression by transfected MSCs after 7 days. (A) FACS analysis using anti-ALP $\mathrm{Ab}$ on hMSCs, cultured in alginate for 7 days either in expansion medium (EM) or osteogenic medium (OM). Mean fluorescence intensity (MFI) represents the average fluorescence signal of 10,000 cells. Cells were either untransfected (-) or transfected with His/BMP-2 plasmid DNA (+) and stained with an anti-ALP antibody or an isotype matched control antibody (C). (B-K) ALP activity staining (red) on cell monolayers after 7 days of culturing. (B,C,D,E) MG-63 cells, (F,G,H) human MSCs, (I,J,K) goat MSCs. Cells were either untransfected and cultured in EM (B,F,I), untransfected and cultured in EM supplemented with BMP-2 protein $(100 \mu \mathrm{g} / \mathrm{ml})(\mathbf{C})$, untransfected and cultured in OM (D,G,J) or transfected and cultured in EM (E,H,K). Pictures are representative of 2 independent experiments performed in duplicate. The scale bars represent $50 \mu \mathrm{m}$. 


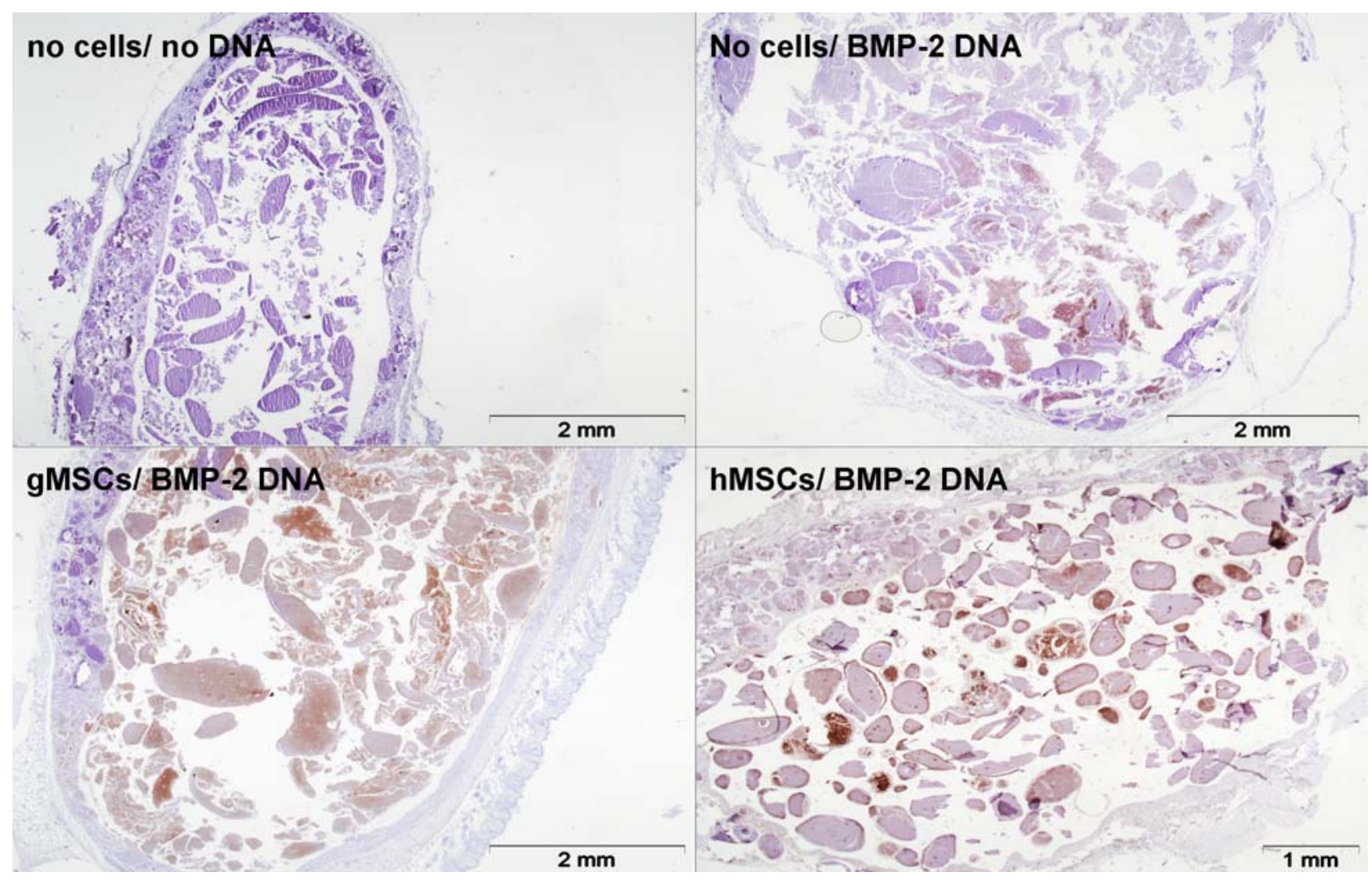

Fig. 5. Osteogenic differentiation in alginate constructs in vivo. Collagen I immunostaining on mouse implants after 6 weeks. Collagen I staining in brown, counterstained with haematoxylin (blue). Representative pictures for each group $(n=6)$ are presented.

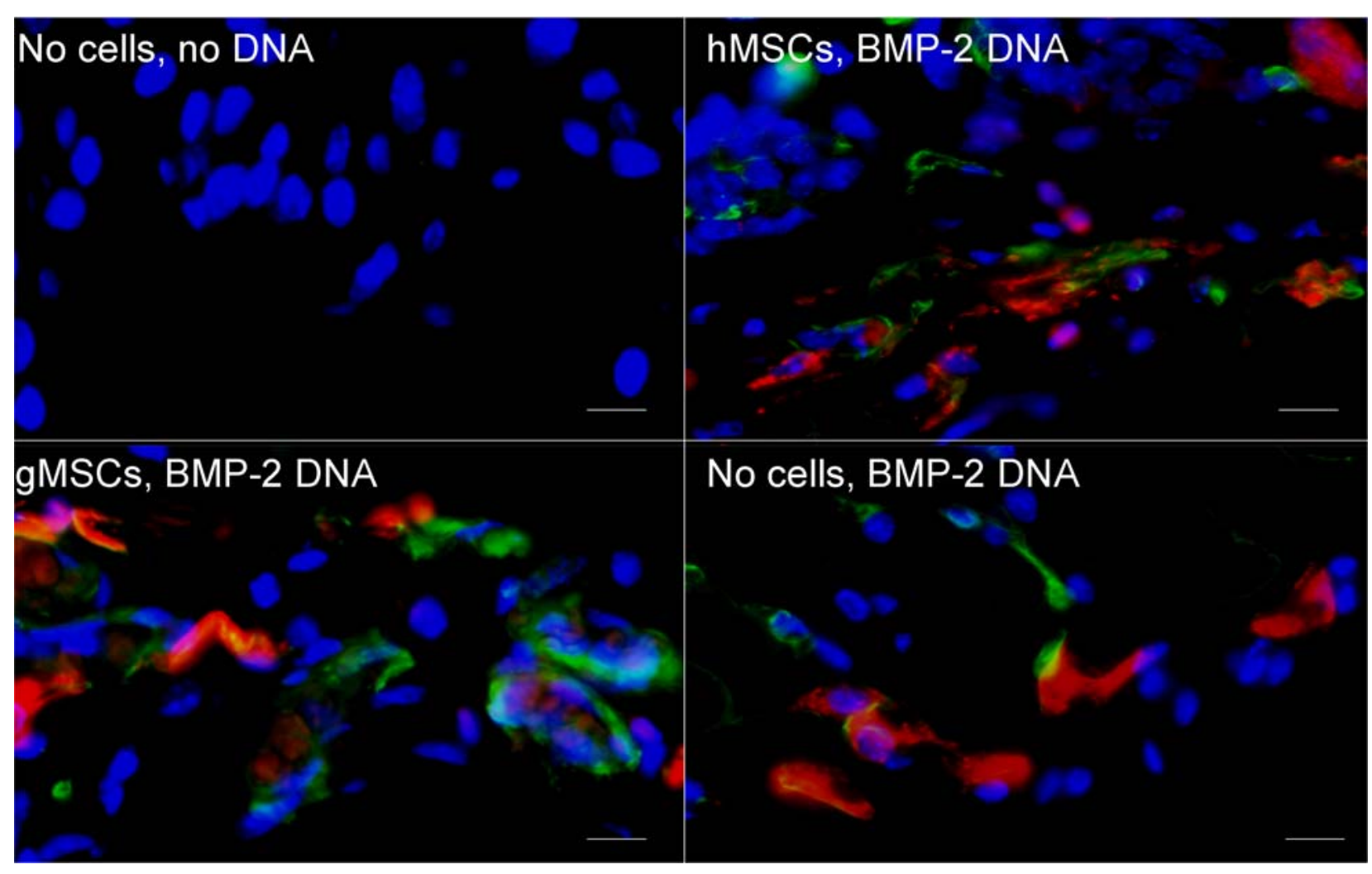

Fig. 6. Paracrine action of His/BMP-2-transfected cells in vivo. Images represent triple immunolocalisation of collagen I in green, anti-His/BMP-2 staining in red, and nuclei in blue. Co-localisation of collagen I and His/BMP2 appears yellow, pictures are representative for 6 implants/group. The scale bars represent $10 \mu \mathrm{m}$. 


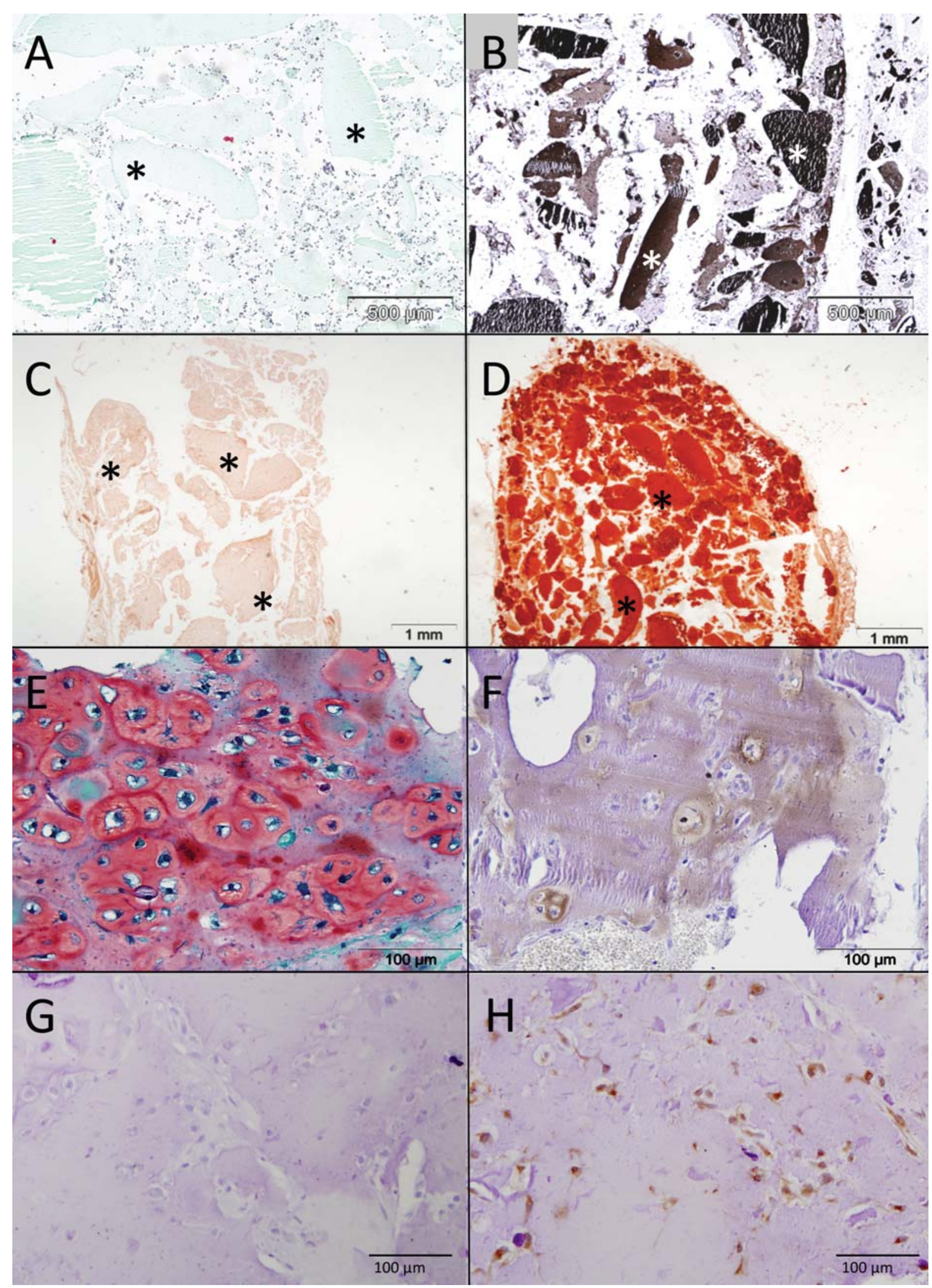

Fig. 7. Alginate mineralisation after in vivo implantation. (A) Goldner's trichrome staining for connective tissue after 6 weeks, collagen appears in green. (B) Von Kossa staining after 6 weeks, mineralisation in black or brown, (C) Alizarin red staining for $\mathrm{Ca}^{2+}$-salts after 2 weeks, calcified tissue in red (hardly present), (D) Alizarin red staining after 6 weeks, calcified tissue in red, (A-D) Pictures are representative for all samples, * indicates alginate patches. (E) Safranin O staining after 6 weeks on hMSC+BMP-2 DNA seeded group, glucosaminoglycans are stained in red, Fast Green counterstaining, (F) Collagen I immunostaining after 6 weeks on hMSC+BMP-2 DNA seeded group. Collagen I in brown, methylene blue counterstaining, (G) Immunolocalisation of osteocalcin after 6 weeks in control group, $(\mathbf{H})$ Osteocalcin staining after 6 weeks on hMSC+BMP-2 DNA seeded group. Positive cells stained brown. 
Transfections using the His/BMP-2 cDNA were similarly performed on MG-63 cells. Immunocytochemistry on cytospun cells transfected with or without lipofectamine-complexed plasmid DNA was performed with an antibody against the His-tag preceding the BMP2 sequence (Fig. 1D) to discriminate between cells producing endogenous BMP-2 and transfected cells. The same cells stained negative using an isotype-matched control antibody (Fig. 1E), whereas untransfected cells also remained unstained (Fig. 1F). MG-63 cells and human and goat MSCs showed high transfection efficiencies, as shown by FACS analysis of His/BMP-2. The efficiency ranged from $64 \%$ up to $95 \%$ with a MFI of 643-713 compared to 38 in the control, consistent in both the liposomecomplexed and the naked DNA group (Fig. 2).

To determine the optimal DNA concentration for BMP2 with and without liposome complexation, hMSCs and MG-63 cells were transfected with different plasmid DNA concentrations ranging from 0.008 to $5 \mu \mathrm{g}$ DNA/gel (100 $\mu \mathrm{l})$. We also performed a FACS analysis of the His-BMP stained cells, which showed little differences in transfection efficiencies and expression levels between all different concentrations (results not shown). Even the lowest concentration tested resulted in significant expression of BMP-2. We also could not find a consistent positive effect of liposome complexation in all concentrations.

To determine BMP-2 levels released by the cells and the duration of production, alginate constructs with His/ BMP-2 plasmid DNA and MG-63 cells (Fig.3) or hMSCs (3 timepoints, results not shown) were cultured for a period up to 5 weeks. The culture medium was collected on a weekly basis and the cultured gels were depolymerised to determine the amount of BMP-2 associated with the gel by ELISA. This revealed a weekly increase of BMP-2 levels in the medium and a much higher weekly increase of BMP-2 in the gel (Fig.3), indicating that the bulk of the produced BMP-2 remained in the gel. The complexation of DNA with liposomes did not have a further additional effect on the amount of released BMP-2.

To evaluate the biological activity of the produced BMP-2, enzymatic stainings on cell monolayers and FACS analyses of gel samples were performed for the early osteogenic marker alkaline phosphatase. Monolayers of MG-63 cells and goat- and human MSCs were transfected with the BMP-2 cDNA and either cultured in expansion medium, or cultured in routine osteogenic differentiation medium to compare the signal. As a negative control, untransfected cells were cultured in expansion medium. In both positive controls and in the BMP-2 transfected group red staining revealed the production of ALP after a week, while the unstimulated cells remained negative (Fig. 4B). Similar results were found for MG-63 cells in gel samples which were analysed by FACS using an anti-ALP antibody. The BMP-2 transfected cells even showed a higher increase in MFI (212) than the positive control, cultured in osteogenic differentiation medium (OM) (MFI=178) compared to untransfected cells cultured in expansion medium (EM) (MFI=72), and the transfected cells stained with an isotype-matched control antibody (MFI=100) (Fig 4A).
To investigate whether transfection in vivo would take place and is able to induce osteogenic differentiation, we implanted gel samples subcutaneously in immunodeficient mice. Analysis of the histological sections after an implantation period of either 2 or 6 weeks showed that in vivo, transfection in alginate was successful as shown by immunohistochemistry on the BMP-2 coupled His-tag. In both the human- and goat MSC seeded groups, numerous positive BMP-2 transfected cells could be found at both the 2 and 6 weeks time points (Fig 1G, H). The samples that received BMP-2 plasmid DNA, but were cell-free, also expressed the transgene (Fig $1 \mathrm{H})$. At 2 weeks, 4/6 samples and at 6 weeks 5/6 samples stained positive for His-tagged BMP-2. This means that resident mouse cells are able to take up the plasmid DNA from the constructs and express it. This proves that transfection in vivo occurs. To determine whether the expressed levels of BMP-2 are sufficient to induce osteogenic differentiation, immunohistochemistry on collagen I was performed (Fig. 5). At two weeks, none of the groups had produced large amounts of collagen I but some staining was visible, mainly in one of the hMSC donors. At 6 weeks, differences between the groups with and without BMP-2 plasmid DNA were far more evident. The groups receiving plasmid DNA showed significantly more collagen I production than the control group without BMP-2 DNA $(p<0.05)$. With the exception of hMSC donor 2, in all the groups in which plasmid DNA was combined with seeded cells, we found the significant highest amount of collagen I staining $(p<0.05)$ compared to non-seeded groups (Fig. 5). To investigate whether the cells expressing the plasmid DNA are also the cells that produce collagen I, or that neighbouring cells are also osteogenically induced, we performed a double staining for both His-tagged BMP-2 and collagen I (Fig. 6). The results show that the His/BMP2 and collagen I staining are present within the same regions in the implants, but only in a few cells co-localise. This suggests that the majority of cells differentiating towards the osteogenic linage do so through a paracrine mechanism (Fig. 6).

To look at alginate morphology changes, HE stained sections were scored for degradation or calcification of the gel, and blood vessel formation. It appears that the gels were slowly degraded, leaving remnants of calcified material, interleaved with ingrown highly vascularised tissue. All of these aspects were found in the implants but occurred independent of the cell type or presence of DNA (Table 1). Alizarin red and von Kossa staining of the samples also confirmed that almost all of the alginate implants calcify between 2 and 6 weeks of implantation, which could contribute to the osteogenic potential of the implants (Fig. 7). The alginate implants also contains connective tissue as shown in green by a Goldner's trichrome staining but no highly cellularised tissue like osteoid, which would be indicated in red (Fig. 7).

At 6 weeks however, cartilaginous tissue is present in the human MSC seeded group as shown by the cell cluster morphology, collagen matrix surrounding the cells and the heavy glycosaminoglycan (GAG) staining, indicated in red by a Safranin O staining (Fig. 7). 
Table 1. Presence of transfected cells and histological parameters in vivo.

\begin{tabular}{|c|c|c|c|c|c|c|c|c|c|c|}
\hline \multirow[b]{2}{*}{ Weeks } & \multicolumn{2}{|c|}{ transfected cells } & \multicolumn{2}{|c|}{ alginate morphology } & \multicolumn{2}{|c|}{ alginate calcification } & \multicolumn{2}{|c|}{ blood vessels } & \multicolumn{2}{|c|}{ collagen I } \\
\hline & 2 & 6 & 2 & 6 & 2 & 6 & 2 & 6 & 2 & 6 \\
\hline no cells/no DNA & 0 & 0 & 7 & 7 & 6 & 8 & 5 & 6 & 4 & 3 \\
\hline no cells/BMP-2 & 6 & 8 & 9 & 7 & 8 & 9 & 3 & 4 & 4 & 6 \\
\hline hMSCs donor 1 & 7 & 8 & 6 & 6 & 9 & 9 & 0 & 3 & 8 & 8 \\
\hline hMSCs donor 2 & 7 & 10 & 7 & 10 & 5 & 10 & 3 & 6 & 5 & 3 \\
\hline gMSCs donor $1^{\star}$ & 4 & 9 & 5 & 6 & 4 & 6 & 3 & 5 & 1 & 10 \\
\hline gMSCs donor 2 & 5 & 4 & 7 & 3 & 7 & 4 & 4 & 6 & 5 & 8 \\
\hline
\end{tabular}

Scoring of transfected cells, alginate properties, blood vessels and collagen $\mathrm{I}$ ( $\mathrm{n}=6$ per group) of the in vivo samples after 2 and 6 weeks, see M\&M for details. Sections were scored by two independent observers. * indicates 1 missing sample.

\section{Discussion and Conclusions}

This study investigates the possibility of creating injectable bone tissue engineering constructs by transfecting cells with BMP-2 plasmid DNA in alginate hydrogel. Both goat and human MSCs and MG-63 cells were found to be capable of efficiently expressing BMP-2 from plasmid DNA and show a sustained release of relevant levels bioactive BMP-2. In vitro, results from MG-63 cells were shown because these are fully differentiated osteoblasts, which is a relevant cell type for bone tissue engineering. Besides MG-63 cells, human MSCs were used because they are one of the most interesting candidates for translation towards a clinical application. Goat MSCs were used because goat is a relevant and widely-used large animal model in bone tissue engineering.

Gene therapy based on non-viral plasmid DNA is pursued by many groups because of reportedly low immunogenicity, transient expression without genetic recombination, and the production of large protein quantities at low cost. However, non-viral gene therapy is also known to have variable and low transfection efficiencies (Lam and Dean, 2010). Most groups transfecting in alginate also report low transfection efficiencies and do not comment on the amount of protein produced by their constructs (Sone et al., 2002; Higashi et al., 2004).

Our results demonstrate that the combination of plasmid DNA with alginate hydrogel however, induces high transfection efficiencies with different constructs, at different DNA concentrations in different cell types. The alginate incorporation might be an important factor in the mechanism behind these successful naked DNA transfections. Two possible explanations for high transfection efficiencies can be considered. The first theory describes DNA uptake via calcium phosphate precipitation. The alginate gels are polymerised by a $100 \mathrm{mM}$ calciumchloride solution. This is a 50-100 times higher concentration of calcium than in the bloodstream or culture medium. Combined with phosphates, calciumphosphate/ DNA co-precipitates might be formed, inducing DNA uptake into the cells (Graham and van der Eb, 1973; Jordan et al., 1996; Chenuet et al., 2008). Macropinocytosis, a different transfection method, transfers naked DNA into the cell, mediated by proteoglycans and other proteins present in culture medium (Wittrup et al., 2007). In the context of alginate, we did not observe a clear additive effect from the use of liposomal transfection agent, even though literature describes this as an even more successful transfection method than the use of calcium phosphate precipitation (Somasundaram et al., 1992). Normally the positively charged liposome complexes make it easier for DNA liposome complexes to access the negatively charged cell membrane (Clements et al., 2007). The sugar chains of alginate however, might neutralise the effect of liposome complexation on the cell membrane. Even though an optimum for both naked and complexed DNA was expected to be within the range of 5 to $0.008 \mu \mathrm{g} / \mathrm{gel}$, titration of plasmid DNA in the context of alginate revealed that the transfection remains successful at both high or very low DNA concentrations.

An increased release of BMP-2 protein production over a period of 5 weeks was found by ELISA analysis. This was a surprising result, considering the transient nature of non-viral transfections (Stiehler et al., 2006). Since not only culture medium samples were collected but BMP-2 levels were also measured in the depolymerised alginate, we found that a large percentage of the produced protein is contained in the gel. This probably creates a release system in which protein is produced by the transfected cells and retained in the gel. Degradation of the gel the protein then mediates its release into the medium over time which could be favourable in vivo since BMP-2 protein in the bloodstream has a half-life of several minutes. Cells might also be able to retain the plasmid DNA for several weeks, especially if little cell division takes place (Hengge et al., 2001; Wolff and Budker, 2005).

When investigating whether osteogenic differentiation could be induced by BMP-2 transfection, the expression of early osteogenic marker ALP both on monolayers and in cells from depolymerised gel was determined. The level of ALP expression in BMP-2 transfected cells cultured in 
EM was similar to that in the positive control group cultured in ODM containing the osteogenic dexamethasone (Duarte et al., 2009). Based on this assay, we conclude that BMP-2 produced from plasmid DNA transfected cells is at least as effective as ODM in directing stem cells into early osteogenic differentiation.

We found that transfection is not only possible in vitro but also in vivo. Transfected cells were found in all groups containing BMP-2 plasmid DNA, either combined with or without human and goat MSCs, at both the 2 and 6 weeks time points. This means that the delivered BMP-2 plasmid DNA is also available for resident cells to take up and produce BMP-2, creating an opportunity to design an off-the-shelf construct without seeded cells. This could overcome several problems such as the risks involved in bone marrow aspirations, or the costs and time involved with culturing stem cells. Similar to the in vitro experiments, osteogenic differentiation also occurs in the in vivo implanted samples, demonstrated by collagen I and osteocalcin staining in the groups containing BMP-2 plasmid DNA, whereas little collagen I and osteocalcin staining is visible in the control group without seeded cells and plasmid DNA. This is in accordance with several studies in which implanted plasmid DNA induces differentiation (Bonadio et al., 1999). This makes it plausible that the induction of collagen I and osteocalcin expression is the result of the BMP-2 transfection. During the implantation period in mice, no bone was formed. This might be explained by the timing of the BMP-2 release, which might be slower compared to other studies (Hosseinkhani et al., 2007; Krebs et al., 2010). A large amount of produced BMP-2 is retained in the alginate and will not be accessible for the cells until the alginate degrades sufficiently. After 6 weeks, we still found patches of alginate present in the implants. It is possible that at later time points bone could be found, since the alginate calcifies between week 2 and week 6 , providing a much more favourable surrounding to deposit bone on for differentiated osteoblasts. In a goat model, in which constructs similar to the ones described here were implanted in the muscle, abundant bone was formed after a longer implantation period (Wegman et al., in preparation).

Remarkably, cartilaginous tissue was formed in the human MSC seeded group. MSCs are known to be able to produce bone via the endochondral bone route, so it would be interesting to see whether the cartilage is eventually mineralised and replaced by bone (Gawlitta et al., 2010).

Another explanation for the lack of bone tissue could be that the concentration of produced BMP-2 is insufficient to induce bone formation. The amount of produced BMP2 , as determined from the in vitro study is in the range of $\mathrm{ng}$, whereas the dosage used in the clinic is in the range of mg (McKay et al., 2007). And even though large doses are used in the clinic, those are considered supraphysiological, and possibly harmful (Shields et al., 2006). Alginate is more and more used as a delivery vehicle for BMPs and turned out to be a good matrix for osteogenic differentiation and reportedly for bone formation (Stevens et al., 2005). As seen in the histological stainings, blood vessel ingrowth necessary for oxygen and nutrient delivery to the cells is efficient, and it calcifies in vivo, creating suitable mineralised matrix for cartilage and eventually bone formation.

Another interesting result is the fact that we found hardly any transfected cells which also produced collagen I. It is possible that other cells surrounding the implants e.g. macrophages take up the plasmid DNA in their attempt to engulf the alginate samples. These cells might then become BMP-2 producing cells, attracting and differentiating nearby osteogenic precursors, but are probably not BMP-2 responsive. This could explain the presence of limited numbers of transfected cells, which are themselves differentiating towards the osteogenic linage.

In summary, the use of gene therapy in bone tissue engineering to deliver osteogenic factors like BMP-2 has several advantages over the use of rhBMP-2 protein. Not only does it overcome the problems of short half-life and instability of BMP-2 resulting in the administration of supra-physiological protein dosages, but in some cases it can also result in more and better bone formation than the administration of rhBMP-2 (Gazit et al., 1999; Kofron and Laurencin, 2006; Pelled et al., 2010).

We developed an economical, efficient non-viral BMP2 gene delivery method by combining alginate with osteogenic cells and BMP-2 plasmid DNA. The alginate hydrogel serves multiple roles as an injectable scaffold, but also functions as a transfection agent and release system, releasing protein for at least 6 weeks. The produced BMP-2 protein has been proven to be biologically active and present at an effective dose to induce early osteogenic differentiation, but not yet bone formation by both human and goat MSCs and MG-63 cells in vitro and in vivo. Based on these results we conclude that after optimisation, this alginate-based gene delivery system might be a promising new development in the field of bone regeneration.

\section{Acknowledgements}

The authors gratefully acknowledge the support of the TeRM Smart Mix Program of the Netherlands Ministry of Economic Affairs and the Netherlands Ministry of Education, Culture and Science and Prof. Y. Tabata (Kyoto University) for the BMP-2 plasmid DNA.

\section{References}

Arthur A, Zannettino A, Gronthos S (2009) The therapeutic applications of multipotential mesenchymal/ stromal stem cells in skeletal tissue repair. J Cell Physiol 218: $237-245$.

Audouy SA, de Leij LF, Hoekstra D, Molema G (2002) In vivo characteristics of cationic liposomes as delivery vectors for gene therapy. Pharm Res 19: 1599-1605.

Bonadio J, Smiley E, Patil P, Goldstein S (1999) Localized, direct plasmid gene delivery in vivo: Prolonged therapy results in reproducible tissue regeneration. Nat Med 5: 753-759. 
Chenuet S, Martinet D, Besuchet-Schmutz N, Wicht M, Jaccard N, Bon AC, Derouazi M, HackerDL, Beckmann JS, Wurm FM (2008) Calcium phosphate transfection generates mammalian recombinant cell lines with higher specific productivity than polyfection. Biotechnol Bioeng 101: 937-945.

Clements BA, Incani V, Kucharski C, Lavasanifar A, Ritchie B, Uludag H (2007) A comparative evaluation of poly-L-lysine-palmitic acid and Lipofectamine 2000 for plasmid delivery to bone marrow stromal cells. Biomaterials 28: 4693-4704.

Dass CR (2004) Lipoplex-mediated delivery of nucleic acids: Factors affecting in vivo transfection. J Mol Med 82: $579-591$.

Duarte AR, Mano JF, Reis RL (2009) Dexamethasoneloaded scaffolds prepared by supercritical assisted phase inversion. Acta Biomater 5: 2054-2062.

Gawlitta D, Farrell E, Malda J, Creemers LB, Alblas J, Dhert WJ (2010) Modulating endochondral ossification of multipotent stromal cells for bone regeneration. Tissue Eng Part B Rev 16: 385-395.

Gazit D, Turgeman G, Kelley P, Wang E, Jalenak M, Zilberman Y, Moutsatsos I (1999) Engineered pluripotent mesenchymal cells integrate and differentiate in regenerating bone: A novel cell-mediated gene therapy. J Gene Med 1: 121-133.

Graham FL, van der Eb AJ (1973) A new technique for the assay of infectivity of human adenovirus 5 DNA. Virology 52: 456-467.

Hengge UR, Dexling B, Mirmohammadsadegh A (2001) Safety and pharmacokinetics of naked plasmid DNA in the skin: Studies on dissemination and ectopic expression. J Invest Dermatol 116: 979-982.

Higashi T, Nagamori E, Sone T, Matsunaga S, Fukui K (2004) A novel transfection method for mammalian cells using calcium alginate microbeads. J Biosci Bioeng 97: 191-195.

Hosseinkhani H, Hosseinkhani M, Khademhosseini A, Kobayashi H (2007) Bone regeneration through controlled release of bone morphogenetic protein-2 from 3-D tissue engineered nano-scaffold. J Control Release 117: 380-386.

Hunt NC, Grover LM (2010) Cell encapsulation using biopolymer gels for regenerative medicine. Biotechnol Lett 32: 733-742.

Jordan M, Schallhorn A, Wurm FM (1996) Transfecting mammalian cells: optimization of critical parameters affecting calcium-phosphate precipitate formation. Nucleic Acids Res 24: 596-601.

Khan Y, Yaszemski MJ, Mikos AG, Laurencin CT (2008) Tissue engineering of bone: material and matrix considerations. J Bone Joint Surg Am 90 Suppl 1: 36-42.

Kofron MD, Laurencin CT (2006) Bone tissue engineering by gene delivery. Adv Drug Deliv Rev 58: 555-576.

Kong HJ, Kim ES, Huang YC, Mooney DJ (2008) Design of biodegradable hydrogel for the local and sustained delivery of angiogenic plasmid DNA. Pharm Res 25: 1230-1238.

Krebs MD, Salter E, Chen E, Sutter KA, Alsberg E (2010) Calcium phosphate-DNA nanoparticle gene delivery from alginate hydrogels induces in vivo osteogenesis. J Biomed Mater Res A 92: 1131-1138.

Lam AP, Dean DA (2010) Progress and prospects: Nuclear import of nonviral vectors. Gene Ther 17: 439447.

Louise C (2006) Nonviral vectors. Methods Mol Biol 333: 201-226.

Luo D, Saltzman WM (2000) Synthetic DNA delivery systems. Nat Biotechnol 18: 33-37.

Ma B, Zhang S, Jiang H, Zhao B, Lv H (2007) Lipoplex morphologies and their influences on transfection efficiency in gene delivery. J Control Release 123: 184194.

McKay WF, Peckham SM, Badura JM (2007) A comprehensive clinical review of recombinant human bone morphogenetic protein-2 (infuse bone graft). Int Orthop 31: 729-734.

Park DJ, Choi JH, Leong KW, Kwon JW, Eun HS (2007) Tissue-engineered bone formation with gene transfer and mesenchymal stem cells in a minimally invasive technique. Laryngoscope 117: 1267-1271.

Pelled G, Ben-Arav A, Hock C, Reynolds DG, Yazici C, Zilberman Y, Gazit Z, Awad H, Gazit D, Schwarz EM (2010) Direct gene therapy for bone regeneration: Gene delivery, animal models, and outcome measures. Tissue Eng Part B Rev 16: 13-20.

Pietersz GA, Tang CK, Apostolopoulos V (2006) Structure and design of polycationic carriers for gene delivery. Mini Rev Med Chem 6: 1285-1298.

Poynton AR, Lane JM (2002) Safety profile for the clinical use of bone morphogenetic proteins in the spine. Spine 27: S40-48.

Riley EH, Lane JM, Urist MR, Lyons KM, Lieberman JR (1996) Bone morphogenetic protein-2: Biology and applications. Clin Orthop Relat Res: 39-46.

Shields LB, Raque GH, Glassman SD, Campbell M, Vitaz T, Harpring J, Shields CB (2006) Adverse effects associated with high-dose recombinant human bone morphogenetic protein-2 use in anterior cervical spine fusion. Spine 31: 542-547.

Shimer AL, Oner FC, Vaccaro AR (2009) Spinal reconstruction and bone morphogenetic proteins: Open questions. Injury 40 Suppl 3: S32-38.

Somasundaram C, Tournier I, Feldmann G, Bernuau D (1992) Increased efficiency of gene transfection in primary cultures of adult rat hepatocytes stimulated to proliferate: A comparative study using the lipofection and the calcium phosphate precipitate methods. Cell Biol Int Rep 16: 653662.

Sone T, Nagamori E, Ikeuchi T, Mizukami A, Takakura Y, Kajiyama S, Fukusaki E, Harashima S, Kobayashi A, Fukui K (2002) A novel gene delivery system in plants with calcium alginate micro-beads. J Biosci Bioeng 94: 87-91.

Southwood LL, Frisbie DD, Kawcak CE, McIlwraith CW (2004) Delivery of growth factors using gene therapy to enhance bone healing. Vet Surg 33: 565-578.

Stevens MM, Marini RP, Schaefer D, Aronson J, Langer R, Shastri VP (2005) In vivo engineering of organs: The bone bioreactor. Proc Natl Acad Sci USA 102: 1145011455. 
Stiehler M, Duch M, Mygind T, Li H, Ulrich-Vinther M, Modin C, Baatrup A, Lind M, Pedersen FS, Bunger CE (2006) Optimizing viral and non-viral gene transfer methods for genetic modification of porcine mesenchymal stem cells. Adv Exp Med Biol 585: 31-48.

Wittrup A, Sandgren S, Lilja J, Bratt C, Gustavsson N, Morgelin M, Belting M (2007) Identification of proteins released by mammalian cells that mediate DNA internalization through proteoglycan-dependent macropinocytosis. J Biol Chem 282:27897-27904.

Wolff JA, Budker V (2005) The mechanism of naked DNA uptake and expression. Adv Genet 54: 3-20.

\section{Discussion with Reviewer}

Reviewer I: What parameter(s) could be optimised to improve the quantity of BMP-2 produced by the efficiently transfected cells?
Authors: Several parameters could be optimised such as cell concentrations in the constructs and degradation of the gel. Faster degradation of the gel could increase the availability of DNA and produced protein for the cells.

Reviewer I: The alginate hydrogel mixed with BMP-2 plasmid only contained $3 \mu \mathrm{g}$ of DNA. Do the authors know the maximum capacity of alginate hydrogel for binding DNA? Could that influence the uptake of the plasmid in vivo, and the overall efficacy of the gene-activated matrix? Authors: The maximum capacity is unknown, but increasing the amount of DNA in the construct does not automatically lead to higher efficiency of the construct (our unpublished results). 